\title{
Aplicação da pressão positiva contínua nas vias aéreas em pacientes em pós-operatório de cirurgia bariátrica
}

\author{
Applying continuous positive airways pressure in patients after bariatric surgery
}

Eli Maria Pazzianotto-Forti', Thaís de Lima Laranjeira ${ }^{2}$, Bruna Gallo da Silva ${ }^{3}$, Maria Imaculada de Lima Montebello ${ }^{4}$ Irineu Rasera Jr. ${ }^{5}$

\begin{abstract}
RESUMO I O objetivo deste trabalho foi avaliar o efeito da aplicação da pressão positiva contínua nas vias aéreas (CPAP), na frequência respiratória (FR), no volume corrente (VC) e no volume minuto (VM), em pacientes em pós-operatório de cirurgia bariátrica. Foram estudadas dez pacientes com média de idade $29,8 \pm 8$ anos, classificadas como obesas mórbidas [índice de massa corpórea (IMC) de $47,5 \pm 7,2 \mathrm{~kg} / \mathrm{m}^{2}$ ] que receberam CPAP de 8 a $10 \mathrm{cmH}_{2} \mathrm{O}$, por 30 minutos, uma vez ao dia, durante dois dias consecutivos, no pós-operatório de cirurgia bariátrica. Antes e após a aplicação da CPAP, foram aferidos a FR e o VM por meio do ventilômetro e, de forma indireta, foi calculado o VC. Para verificar a normalidade dos dados foi aplicado o teste de Shapiro-Wilk, e, após, as medidas foram comparadas com o uso do Teste $t$ de Student e de Man-Whitney. As análises foram processadas com o uso do SPSS 7,5 considerando o nível de 5\% de significância. Houve um aumento significativo para as seguintes variáveis estudadas no primeiro e no segundo dia de aplicação: $F R=20 \pm 6$ resp/ min versus $26 \pm 7 \mathrm{resp} / \mathrm{min}(\mathrm{p}=0,009)$ e $\mathrm{FR}=22 \pm 7 \mathrm{resp} /$ min versus $26 \pm 8 \mathrm{resp} / \mathrm{min}$ ( $\mathrm{p}=0,007$ ); $\mathrm{VM}=9,57 \pm 2,75$ $L$ versus $12,39 \pm 4,18 L(p=0,041)$ e VM=9,71 $\pm 2,52 L$ versus $11,18 \pm 2,96 \mathrm{~L}(\mathrm{p}=0,037)$. Os valores do $\mathrm{VC}=360 \pm 157,59 \mathrm{~mL}$ versus $440 \pm 69,18 \mathrm{~mL}(\mathrm{p}=0,21)$ e $401 \pm 90,46 \mathrm{~mL}$ versus $416 \pm 78,04 \mathrm{~mL}(p=0,18)$ não apresentaram diferença significativa na comparação pré- e pós-aplicação, nos dois
\end{abstract}

\begin{abstract}
I The objective was to evaluate the effect of continuous positive airway pressure (CPAP) in the respiratory rate (RR), tidal volume (TV) and minute volume (MV) in patients after bariatric surgery. Ten patients with mean age $29.8 \pm 8$ years, classified as morbidly obese (BMI $47.5 \pm 7.2 \mathrm{~kg} / \mathrm{m}^{2}$ ) who received CPAP from 8 to $10 \mathrm{cmH}_{2} \mathrm{O}$, for 30 minutes, once a day for two consecutive days, in the post-bariatric surgery were studied. Before and after applying CPAP were measured RR and the MV through the ventilometer, and indirectly the TV were calculated. The Shapito-Wilk was applied to check the normality of data, and then the measures were compared using the Student's t-test and Mann-Whitney test. Analyses were processed using the SPSS 7.5 considering the $5 \%$ level of significance. There was a significant increase for the following variables in the first and second days of application: $\mathrm{RR}=2 \mathrm{O} \pm 6$ breathing/min versus $26 \pm 7$ breathing/min ( $p=0.009$ ), and $\mathrm{RR}=22 \pm 7$ breathing/min versus $26 \pm 8$ breathing $/ \mathrm{min}$ $(p=0,007) ; M V=9.57 \pm 2.75 L$ versus $12.39 \pm 4.18 L(p=0.041)$, and $M V=9.71 \pm 2.52 \mathrm{~L}$ versus $11,18 \pm 2.96 \mathrm{~L}(\mathrm{p}=0.037$ ). The values of $T V=360 \pm 157,59 \mathrm{~mL}$ versus $440 \pm 69,18 \mathrm{~mL}(\mathrm{p}=0,21)$, and $401 \pm 90,46 \mathrm{~mL}$ versus $416 \pm 78,04 \mathrm{~mL}(\mathrm{p}=0,18)$ did not differ significantly when comparing pre- and post-application in two days therapy. Thus, it was concluded that CPAP can be applied as an auxiliary respiratory therapy in the treatment of patients in post-bariatric surgery for the
\end{abstract}

Estudo desenvolvido nos Cursos de Graduação e Mestrado em Fisioterapia da Faculdade de Ciências da Saúde da Universidade Metodista de Piracicaba (UNIMEP) - São Paulo (SP), Brasil e no Hospital dos Fornecedores de Cana de Piracicaba (HFC) - São Paulo (SP), Brasil.

'Professora Doutora do Curso de Fisioterapia do Programa de Mestrado em Fisioterapia e do Curso de Especialização em Fisioterapia Cardiorrespiratória da UNIMEP - Piracicaba (SP), Brasil.

2Fisioterapeuta; Programa de Pós-graduação da UNIMEP - Piracicaba (SP), Brasil.

${ }^{3}$ Programa de Pós-graduação (Mestrado) em Fisioterapia da UNIMEP - Piracicaba (SP), Brasil.

${ }^{4}$ Professora Doutora da Faculdade de Ciências Matemáticas e da Natureza da UNIMEP - Piracicaba (SP), Brasil; Professora-Colaboradora do Programa de Mestrado em Fisioterapia da UNIMEP - Piracicaba (SP), Brasil.

${ }^{4}$ Médico Especialista em Gastrocirurgia; Diretor da Clínica Bariátrica de Piracicaba - Piracicaba (SP), Brasil. 
dias de terapia. Assim, foi possível concluir que a CPAP pode ser aplicada como recurso auxiliar da fisioterapia respiratória no tratamento de pacientes em período pós-operatório de cirurgia bariátrica, para a manutenção do VC, porém atenção deve ser tomada durante a aplicação, pois pode haver aumento da FR.

Descrittores | obesidade mórbida; cirurgia bariátrica; pressão positiva contínua nas vias aéreas; fisioterapia.

\section{INTRODUÇÃO}

A obesidade é uma doença que afeta as nações desenvolvidas e em desenvolvimento, sendo considerada uma epidemia mundial que atinge indivíduos em todos os níveis sócioeconômicos ${ }^{1,2}$. Pode ser definida como um acúmulo excessivo de gordura corporal, em extensão tal, que acarreta prejuízos à saúde, tais como alterações na função respiratória, distúrbios dermatológicos e do aparelho locomotor, além de favorecer o surgimento de enfermidades potencialmente letais, como dislipidemias, doenças cardiovasculares, diabetes tipo II e certos tipos de câncer ${ }^{2,3}$.

A cirurgia bariátrica é o método mais efetivo para o tratamento de doentes com obesidade grave, pois produz perda de peso significativa. As cirurgias que apresentam melhores resultados podem reduzir o peso entre 30 e $40 \%$ e esse efeito pode ser mantido por longos períodos ${ }^{4,5}$.

Assim, como todo procedimento cirúrgico especialmente do andar superior do abdome, a cirurgia bariátrica produz efeitos deletérios no sistema respiratório, como alterações na troca gasosa e na mecânica respiratória. O tempo cirúrgico e os anestésicos empregados acarretam maior chance de ocorrência de complicações pulmonares pós-operatórias ${ }^{6}$. Nos obesos esses efeitos são ainda mais evidentes, tornando-os mais suscetíveis a essas complicações, e predispondo-os ao desenvolvimento de atelectasias no período pós-operatório ${ }^{7}$. Comparativamente com a população em geral, os indivíduos obesos apresentam maior probabilidade de desencadearem atelectasias, aproximadamente $45 \%$, no pós-operatório ${ }^{6}$. Além de a obesidade acarretar alterações nos volumes e capacidades pulmonares, indivíduos submetidos à gastroplastia também apresentam alterações da força muscular respiratória ${ }^{8}$ e alterações na troca gasosa, resultando em hipoxemia ${ }^{9,10}$.

Tenório et al. ${ }^{11}$ relataram que a fisioterapia respiratória pré- e pós-operatória em obesos mórbidos é maintenance of TV, but care should be taken during application, there may be increased RR.

Keywords I morbid obesity; bariatric surgery; continuous positive airway pressure; physical therapy specialty.

benéfica e de fundamental importância, independente da técnica empregada. Porém, alguns estudos têm sido realizados, no sentido de investigar recursos da fisioterapia respiratória que tragam contribuição aos pacientes obesos mórbidos durante o período pós-operatório, buscando melhora ou preservação dos volumes, capacidades pulmonares e da mobilidade diafragmática e tóracoabdominal ${ }^{12,13}$.

Entretanto, mais estudos devem ser realizados no sentido de se identificar não só o recurso mais eficaz mas, também, o melhor momento para a aplicação, o tempo de aplicação, a intensidade, entre outros.

Neste sentido, a pressão positiva contínua nas vias aéreas (CPAP) é um recurso terapêutico empregado na fisioterapia respiratória, a qual tem a função de evitar o colapso das vias aéreas superiores. Ela promove o aumento dos estoques corpóreos de $\mathrm{O}_{2}$ mediante o incremento da capacidade residual funcional. Ao pressurizar a via aérea reduz a instabilidade respiratória responsável pelas variações na troca gasosa e nos volumes e capacidades pulmonares. As alterações pulmonares presentes no pós-operatório persistem por algum tempo, o que predispõe os pacientes às complicações ${ }^{14}$. A fisioterapia respiratória com a utilização da CPAP tem por finalidade prevenir ou minimizar as alterações pulmonares comuns no pós-operatório, restaurando volumes e capacidades pulmonares ${ }^{7}$.

O objetivo do presente estudo foi avaliar o efeito do método CPAP sobre a frequência respiratória (FR), o volume corrente (VC) e o volume minuto (VM) no pós-operatório de cirurgia bariátrica.

\section{MATERIAL E MÉTODOS}

Participaram dessa série de casos 10 pacientes do sexo feminino, com média de idade $29,8 \pm 8$ anos e índice de massa corpórea (IMC) de 47,5 $\pm 7,2 \mathrm{~kg} / \mathrm{m}^{2}$. Os critérios 
de inclusão foram: obesas mórbidas (IMC $\geq 40 \mathrm{~kg} / \mathrm{m}^{2}$ ), não tabagistas, com ausência de doenças pulmonares, cardíacas e tromboembólicas, submetidas à cirurgia bariátrica por laparotomia pela técnica do bypass gástrico em Y de Roux. Foram excluídas as voluntárias que apresentaram instabilidade clínica no pós-operatório, as que necessitaram de internação em terapia intensiva, as que se negaram a participar do estudo, as que não conseguiram realizar as atividades propostas pelo pesquisador por falta de entendimento ou apresentaram intolerância à máscara facial.

Respeitando as normas de condutas em pesquisa experimental com seres humanos, este estudo foi desenvolvido seguindo as orientações do conselho nacional de saúde, resolução 196/96. Após ter sido aprovado pelo Comitê de Ética da instituição sob o parecer no 30/09.

As voluntárias foram informadas e esclarecidas a respeito dos objetivos e da metodologia experimental as quais seriam submetidas, sendo explicitado o caráter não invasivo dos procedimentos.

Para a realização da CPAP foi utilizado um gerador de fluxo com uma fração inspirada de oxigênio ajustada para liberar um fluxo de mais de $140 \mathrm{~L} / \mathrm{min}$ (Whisperflow, Caradyne, Ireland). Por meio de uma traqueia corrugada, o gerador de fluxo foi conectado à máscara facial siliconizada, com válvula unidirecional, sendo que no ramo expiratório da válvula, foi colocada uma válvula de pressão positiva expiratória final (PEEP) em $\mathrm{cmH}_{2} 0$ do tipo spring-loaded (Vital Signs Inc, Totoma, NJ). A pressão positiva contínua ofertada às voluntárias foi o somatório da pressurização oferecida pela válvula de PEEP e do fluxo liberado pelo equipamento, que permaneceu entre 8 e $10 \mathrm{cmH}_{2} \mathrm{O}$, respeitando o conforto da paciente. A máscara foi acoplada à face da paciente por fixadores de borracha e a monitorização durante a aplicação foi realizada de forma contínua pelo terapeuta.

Para a avaliação da FR e do VM foi utilizado um ventilômetro analógico $\mathrm{OHMEDA}{ }^{\circledR}$, com registro em litros, acoplado a uma máscara facial simples. O cálculo do VC foi realizado de forma indireta, por meio do cálculo: VM/FR.

\section{Procedimentos}

Todas as pacientes avaliadas apresentaram curso evolutivo pós-operatório dentro da normalidade, e receberam alta do hospital no tempo previsto que foi de três dias.

As pacientes receberam a intervenção da CPAP uma vez ao dia, durante 30 minutos, durante dois dias, ou seja, no primeiro dia após a cirurgia $\left(\mathrm{PO}_{1}\right)$ e no segundo dia após a cirurgia $\left(\mathrm{PO}_{2}\right)$; com um nível de PEEP entre 8 e $10 \mathrm{cmH}_{2} \mathrm{O}$, posicionadas em fowler (45\%).

Antes e após a aplicação do protocolo, foram aferidas a frequência cardíaca, a pressão arterial e três medidas da FR e do VM com intervalos de cinco minutos entre elas. As medidas de volume corrente foram obtidas dividindo-se os valores do VM pela FR. As medidas foram obtidas com as voluntárias posicionadas em Fowler (45). A escolha dessa posição se deve à padronização para a realização tanto das avaliações como do tratamento, uma vez que, para a maioria das voluntárias, o conforto maior para a permanência durante o período pós-operatório é no leito hospitalar com a cabeceira elevada. As medidas foram realizadas três vezes ou até que elas apresentassem valores próximos entre si. Para o cálculo final, foram registradas as medidas de maior valor.

As avaliações no pós-operatório foram realizadas após a aplicação da escala visual analógica da dor, a qual varia de 0, nenhuma dor, a 10, dor intensa. Quando os valores obtidos foram maiores do que quatro na escala, as voluntárias receberam administração de analgésicos antes da avaliação.

\section{ANÁLISE ESTATÍSTICA}

As análises foram processadas com o uso do software SPSS 7.5, considerando o nível de 5\% de significância. As variáveis FR, VM e VC foram tomadas antes e após a aplicação da CPAP, no primeiro e no segundo pós-operatórios, e foram apresentadas em média e desvio-padrão. Para verificar a normalidade dos dados foi aplicado o teste de Shapiro-Wilk e uma vez constatada a normalidade ou não dos dados, as medidas tomadas antes e após a CPAP foram comparadas com o uso do teste $t$ de Student ou o teste de Mann-Whitney.

O tamanho da amostra apresentou um power de $80 \%$ com nível de significância de 5\% e teve o VC como a principal variável.

\section{RESULTADOS}

Neste estudo foram avaliadas e tratadas 10 voluntárias com idade de 29,8 \pm 8 anos, massa corporal de $125,35 \pm 22 \mathrm{~kg}$, altura de $1,61 \pm 0,05 \mathrm{~m}$ e IMC de $47,5 \pm 7,2 \mathrm{~kg} / \mathrm{m}^{2}$. As voluntárias foram submetidas à 
Tabela1. Idade e características antropométricas das voluntárias estudadas

$\begin{array}{lc} & \text { CPAP }(\mathrm{n}=10) \\ \text { Idade (anos) } & 29,8 \pm 8 \\ \text { Estatura }(\mathrm{m}) & 1,61 \pm 0,05 \\ \text { Massa Corporal }(\mathrm{kg}) & 125,35 \pm 22 \\ \text { IMC (kg/m²) } & 47.5 \pm 7.2 \\ \text { Tempo de anestesia (min) } & 104 \pm 7,6 \\ \text { Tempo de ventilação mecânica (min) } & 130,4 \pm 8,6 \\ \text { Tempo de internação (dias) } & 3\end{array}$

CPAP: pressão positiva contínua nas vias aéreas; IMC: índice de massa corpórea

Tabela 2. Valores médios, desvios-padrões e resultados estatísticos das variáveis estudadas no primeiro dia pós-operatório $\left(\mathrm{PO}_{1}\right)$ e no segundo dia pós-operatório $\left(\mathrm{PO}_{2}\right)$ pré- e pós-aplicação da pressão positiva contínua nas vias aéreas $(n=10)$

\begin{tabular}{lcccc}
\multirow{2}{*}{ Variáveis } & \multicolumn{2}{c}{$\mathrm{PO}_{1}-\mathrm{CPAP}$} & \multicolumn{2}{c}{$\mathrm{PO}_{2}-\mathrm{CPAP}$} \\
\cline { 2 - 5 } & Antes & Após & Antes & Após \\
FR (rpm) & $20 \pm 6$ & $26 \pm 7^{*}$ & $22 \pm 7$ & $26 \pm 8^{*}$ \\
VM $(\mathrm{L})$ & $9,57 \pm 2,71$ & $12,39 \pm 4,18^{*}$ & $9,71 \pm 2,52$ & $11,18 \pm 2,96^{*}$ \\
VC $(\mathrm{mL})$ & $360 \pm 157,59$ & $440 \pm 69,18$ & $401 \pm 90,46$ & $416,19 \pm 78,04$
\end{tabular}

PO: pós-operatório; FR: frequência respiratória; VM: volume minuto; VC: volume corrente; CPAP: pressão positiva contínua nas vias aéreas

*diferença estatística $p<0,05$ entre pré- e pós-aplicação da CPAP

cirurgia bariátrica por via laparotomia, tiveram tempo de ventilação mecânica de 130,4 $\pm 8,65$ min, de anestesia de $104 \pm 7,6$ min e permaneceram três dias no hospital, conforme mostra a Tabela 1 . O tempo de ventilação mecânica corresponde ao tempo entre a intubação oro-traqueal e a extubação, e o tempo de anestesia corresponde ao tempo entre a indução anestésica e a extubação.

Quando avaliadas as medidas da FR antes e após a aplicação da CPAP nos dois dias estudados ( $\mathrm{p}=0,009$ e $\mathrm{p}=0,007)$, observa-se que há diferença estatística significativa para as respectivas medidas nos dois momentos.

Da mesma forma, as medidas de VM para os momentos antes e após a aplicação da CPAP nos dois dias estudados também evidenciaram uma diferença significativa ( $p=0,041$ e $p=0,037)$.

As medidas de VC não apresentaram diferença significativa em comparação pré- e pós-aplicação da CPAP ( $\mathrm{p}=0,21$ e $\mathrm{p}=0,18)$ quando comparadas no $\mathrm{PO}_{1}$ e no $\mathrm{PO}_{2}$.

A Tabela 2 descreve os resultados obtidos para as medidas de FR, VC e VM observadas, considerando o $\mathrm{PO}_{1}$ e o $\mathrm{PO}_{2}$.

\section{DISCUSSÃO}

Este estudo foi realizado a fim de verificar a eficácia da aplicação da CPAP em pacientes obesos mórbidos no período pós-operatório de cirurgia bariátrica, no que se refere aos volumes pulmonares e frequência respiratória. Pôde-se constatar que a aplicação da CPAP promoveu a manutenção do $\mathrm{VC}$, mas pode ter influenciado no aumento da FR e assim do VM.

Alguns estudos demonstram que o uso da CPAP reduz a necessidade de intubação, o tempo de permanência na UTI, a incidência de pneumonias, infecções, sepse e o desenvolvimento de hipoxemia aguda após cirurgia abdominal eletiva de grande porte, em função da restauração dos volumes e capacidades pulmonares ${ }^{9,11}$. De acordo com os resultados obtidos no presente estudo, pôde-se constatar tal restauração em relação ao volume corrente, o que pode ser considerado um benefício ao portador de obesidade e especialmente ao paciente submetido à cirurgia bariátrica, uma vez que a literatura aponta redução de volumes e capacidades em função da disfunção diafragmática ${ }^{8}$.

Nessa situação, ocorre tendência a diminuição do volume corrente e o desencadeamento de uma respiração rápida e superficial para a manutenção do volume-minuto suficiente para a preservação das trocas gasosas ${ }^{11}$. No presente estudo, embora tenha havido a manutenção do VC, pode-se constatar o aumento da FR e, consequentemente, do VM. Isso pode ser explicado talvez por uma inadequada adaptação à máscara facial ou pela dificuldade de ajuste do sistema de CPAP utilizado. Pois, segundo a literatura, a aplicação da CPAP busca restaurar os volumes comprometidos, diminuir a frequência respiratória, promovendo mais conforto respiratório ao paciente, diminuindo o volume-minuto, entretanto, levando a uma normalização do volume corrente, diminuído em função do padrão ventilatório assumido no pós-operatório de cirurgia abdominal ${ }^{9}$.

Segundo Barbalho-Moulin et al., ${ }^{15}$ a gastroplastia, por se tratar de uma cirurgia abdominal alta, apresenta alterações inerentes a esse procedimento, tais como: redução dos volumes pulmonares, aumento da frequência respiratória em função da disfunção da musculatura respiratória que, por sua vez, leva ao comprometimento do mecanismo da tosse, que é fundamental para a promoção da higiene brônquica neste período ${ }^{16,17}$.

Assim, verificou-se que a aplicação da CPAP promoveu manutenção do volume corrente quando aplicado no pós-operatório da gastroplastia tanto no $\mathrm{PO}_{1}$ quanto no $\mathrm{PO}_{2}$. Isso ficou evidenciado pelo fato de as medidas do VC não terem mostrado diferença significativa, na comparação pré- e pós-aplicação da CPAP no $\mathrm{PO}_{1}$ e no $\mathrm{PO}_{2}$, o que sinaliza efeito benéfico, pois aponta manutenção dos volumes pulmonares. 
Entretanto, o VM demonstrou significativo aumento após a aplicação da CPAP, nos dois dias de tratamento. Tal incremento provavelmente se deve ao aumento da FR. O aumento significativo da FR após a aplicação da CPAP, na comparação pré- e pós-aplicação, está de acordo com o preconizado por Muller et al., ${ }^{18}$ que contrapõem a recomendação da utilização de CPAP com o objetivo de diminuir o trabalho respiratório em pacientes em pós-operatórios. Atribui o aumento do trabalho respiratório a aplicação de altas taxas de pressão nas vias aéreas, resultando em um maior desconforto durante a inspiração e esforço do paciente durante a expiração que, além de ser atribuído aos altos fluxos, também pode estar associado a um aumento da resistência expiratória imposta pela (PEEP), promovendo um aumento da frequência respiratória.

Entretanto Gaszynski ${ }^{19}$ considera aumento do trabalho respiratório sinais como retração substernal, utilização da musculatura acessória da inspiração e aparecimento da respiração paradoxal, sinais esses que não foram detectados neste estudo.

Por um lado, no presente estudo, mesmo tomando os devidos cuidados durante a aplicação da terapia, não pôde ser descartada a possibilidade de alterações nos ajustes do fluxo, ou da válvula de PEEP, ou até mesmo da máscara, no momento pré-aplicação da CPAP, e, assim, esses fatores terem propiciado o aumento da FR e, consequentemente, o do VM.

Por outro, para Schettino et al. ${ }^{16}$ o aumento do VM após a aplicação do protocolo CPAP pode ser verificado, pois o equipamento promove maior recrutamento alveolar através da PEEP, evitando atelectasias e sendo considerado uma das formas efetivas para tratar a hipoventilação. No presente estudo, não se pode afirmar que houve recrutamento alveolar pois, embora o VM tenha aumentado, houve manutenção do VC e, então, somente se pode atribuir ao aumento do VM, o aumento da FR.

Segundo Gaszynski ${ }^{19}$, os pacientes devem não só aprender a respirar dentro da máscara como também tolerá-la e, atribui-se a isso o fato do aparecimento da sensação de dispneia e certo desconforto no início da aplicação. Embora não tenha sido avaliada a sensação de dispneia nos pacientes estudados, pode-se constatar aumento da frequência respiratória, o que também pode estar associada à adaptação das pacientes à máscara.

Como limitações do estudo, ressalta-se a falta de exame espirométrico à beira do leito, o qual permitiria discutir resultados de outros volumes pulmonares que sabidamente são relevantes em obesos mórbidos.

\section{CONCLUSÃO}

Por meio deste estudo pôde-se constatar que a aplicação da CPAP promoveu um aumento da FR e do VM e, ao mesmo tempo proporcionou preservação do VC.

Conclui-se que o protocolo da CPAP pode ser aplicado como auxílio da fisioterapia respiratória no tratamento de pacientes em período pós-operatório de cirurgia bariátrica, para a manutenção do $\mathrm{VC}$, porém a atenção deve ser tomada durante a aplicação, porque pode haver aumento da frequência respiratória e, com isso, maior trabalho respiratório.

\section{REFERÊNCIAS}

1 Teichmann L, Olinto MTA, Costa JSD, Ziegler D. Fatores de risco associados ao sobrepeso e à obesidade em mulheres de São Leopoldo, RS. Rev Bras Epidemiol. 2006;9(3):360-73.

2 Coutinho W. Etiologia da obesidade. Rev ABESO. [Internet]. 2007 [cited 2008 Dec 20]; 7(30):14 p. Available from: http://www.abeso.org.br/pdf/ Etiologia\%2Oe\%2OFisiopatologia\%20-\%2OWalmir\%2OCoutinho.pdf

3 Pinheiro ARO, Freitas SFT, Corso ACT. Uma abordagem epidemiológica da obesidade. Rev Nutr. 2004;17(4):523-33.

4 Pareja JC, Pilla VF, Geloneze BN. Operational mechanisms of antiobesity surgeries. Einstein. 2006;4(1):S12O-S4.

5 Anderi JRE, Araujo LGC, Fuhro FE, Godinho CA, Henriques AC. Experiência inicial do serviço de cirurgia bariátrica da Faculdade de Medicina do ABC. Arq Med ABC. 2007:32(1):25-9.

6 Lorentz MN, Albergaria VF, Lima FAZ. Anestesia para obesidade mórbida. Rev Bras Anestesiol. 2007;57(2):199-213.

7 Sanches GD, Gazoni FM, Konishi RK, Guimarães HP, Vendrame LS, Lopes RD. Intensive care of postoperative patients in bariatric surgery. Rev bras ter intensiva. 2007:19(2):205-9

8 Paisani DM, Chiavegato LD, Faresin SM. Volumes, capacidades pulmonares e força muscular respiratória no pós-operatório de gastroplastia. J Bras Pneumol. 2005;31(2):125-32.

9 Squadrone V, Coha M, Cerutti E, Schellino MM, Belloni G, Vilianis G, et al. Continuos positive airway pressure for treatment of postoperative hypoxemia: a randomized controlled trial. JAMA. 2005;293:589-95.

10 Johnston C, Carvalho WB. Pressão positiva contínua em vias aéreas para o tratamento de hipoxemia no pós-operatório. Rev Assoc Med Bras. 2006;52(2):67.

11 Tenório LHS, Lima AMJ, Brasileiro-Santos MS. Intervenção da fisioterapia respiratória na função pulmonar de indivíduos obesos submetidos à cirurgia bariátrica. Uma revisão. Rev Port Pneumol. 2010;16(2):307-14.

12 Forti EMP, Ike D, Barbalho-Moulim M, Rasera Jr I, Costa D. Effects of chest physiotherapy on the respiratory function of postoperative gastroplasty patients. Clinics. 2009;64(7):683-9.

13 Barbalho-Moulim MC, Miguel GPS, Forti EMP, Costa D. Comparação entre inspirometria de incentivo e pressão positiva expiratória na função pulmonar após cirurgia bariátrica. Fisioter Pesq. 2009:16(2):166-72 
14 Freitas IGA, Togeiro SMGP, Tufik S. Tratamento da respiração Cheyne Stokes em pacientes com insuficiência cardíaca congestiva. Arq Bras Cardiol. 2007;88(3):66-70.

15 Barbalho-Moulim MC, Miguel GPS, Forti EMP, César MC, Azevedo JLMC, Costa D. Silicone-ring Roux-en-Y gastric bypass in the treatment of obesity: effects of laparoscopic versus laparotomic surgery on respiration. Obes Surg. 2011;21(2):194-9.

16 Schettino GPP, Reis MAS, Galas F, Park M, Franca S, okamoto V. Ventilação mecânica não-invasiva com pressão positiva. J Bras Pneumol. 2007:19(2):S92-S105.
17 Ramos GC, Gabriel Neto S, Oliveira EC. Avaliação da função pulmonar após colecistectomias laparoscópicas e convencionais. Rev Col Bras Cir. 2007;34(5):326-30.

18 Muller AP, Olandoski M, Macedo RM, Constantino CLCGS. Estudo comparativo entre a pressão positiva intermitente (reanimador Muller) e contínua no pós-operatório de cirurgia de revascularização do miocárdio. Arq Bras Cardiol. 2006;86(3):232-9.

19 Gaszynski T, Tokarz A, Piotrowski D, Machala W. Boussignac CPAP in the Postoperative Period in Morbidly Obese Patients. Obes Surg. 2007:17:452-6. 